\title{
Königsberg à Kaliningrad : problèmes de patrimoines historique et culturel
}

\section{Valeriy Galtsov}

Traducteur : Alexandre Smirnov

\section{Q OpenEdition \\ Journals}

\section{Édition électronique}

URL : https://journals.openedition.org/rbnu/2829

DOI : $10.4000 /$ rbnu.2829

ISSN : 2679-6104

\section{Éditeur}

Bibliothèque nationale et universitaire de Strasbourg

\section{Édition imprimée}

Date de publication : 1 mai 2012

Pagination : 39-45

ISSN : 2109-2761

\section{Référence électronique}

Valeriy Galtsov, «Königsberg à Kaliningrad : problèmes de patrimoines historique et culturel », La Revue de la BNU [En ligne], 5 | 2012, mis en ligne le 01 mai 2012, consulté le 25 août 2021. URL : http:// journals.openedition.org/rbnu/2829; DOI : https://doi.org/10.4000/rbnu.2829

Ce document a été généré automatiquement le 25 août 2021.

\section{cc) (†) (2)}

La Revue de la BNU est mise à disposition selon les termes de la Licence Creative Commons Attribution - Pas d'Utilisation Commerciale - Partage dans les Mêmes Conditions 4.0 International. 


\title{
Königsberg à Kaliningrad : problèmes de patrimoines historique et culturel
}

\author{
Valeriy Galtsov
}

Traduction : Alexandre Smirnov

1 En 2005, on a célébré avec solennité les 750 ans de la ville de Kaliningrad. L'événement était classé dans le registre des manifestations publiques nationales, et pour cette raison le président Poutine a pris part aux festivités.

Parmi les nombreux problèmes préparatoires se posait celui de la dénomination de la ville : quel anniversaire allait-on célébrer au juste, celui de Königsberg-Kaliningrad ou celui de Kaliningrad tout court? Autrement dit, il s'agissait de répondre à une question essentielle: l'histoire de la Kaliningrad russe s'inscrit-elle dans le prolongement de l'histoire de la Königsberg allemande? En apparence, le fait même de célébrer cet anniversaire donnait à penser que oui. Mais en réalité tout n'était pas si simple : le nom de la fête officiellement validé à Moscou était «l'anniversaire des 750 ans de Kaliningrad ", mais dans les médias et dans l'opinion publique on parlait souvent de "l'anniversaire de Königsberg-Kaliningrad" ou on disait de façon évasive «l'anniversaire de la ville ». On faisait donc remarquer par là qu'un lien historique entre les deux villes existait malgré tout. Ainsi on peut légitimement se demander dans quelle mesure Königsberg reste présente à Kaliningrad et s'interroger sur les conséquences de cette situation singulière dans la prise en compte de son patrimoine historique et culturel.

3 L'histoire de Königsberg s'est achevée en avril 1945, quand les troupes de l'armée rouge sont entrées dans la ville. En août 1945, la partie nord-est de la Prusse-Orientale (un tiers du territoire) a été transmise et incorporée à l'Union soviétique par la décision de la conférence de Potsdam (les deux tiers du territoire de la Prusse-Orientale ont été annexés à la Pologne). En 1947-1948 les derniers habitants de Königsberg (selon les statistiques officielles plus de 102000 personnes) ont été déportés en Allemagne. 
4 Königsberg ne figurait pas parmi les villes les plus anciennes de l'Europe ni ne se distinguait par son aspect architectural ou par des monuments uniques. Mais le passé de la ville était riche en personnalités éminentes et son histoire avait une grande importance pour toute l'Allemagne.

5 En 1255 les chevaliers de l'ordre teutonique ont commencé à construire une forteresse sur une colline à l'embouchure du fleuve Pregel. Cette forteresse était un avant-poste destiné à christianiser les Prussiens (en réalité à la conquête violente de leurs terres). $\mathrm{Au} 13^{\mathrm{e}}$ siècle les Prussiens, exterminés en grande partie, n'existaient plus en tant que peuple indépendant. $\mathrm{Au} 14^{\mathrm{e}}$ siècle leurs terres étaient peuplées par des colons allemands venus de Basse-Saxe, de Westphalie, de Poméranie, du Mecklembourg ou de Silésie. À partir du $16^{\mathrm{e}}$ siècle, la Prusse devint une nouvelle patrie pour plusieurs milliers de colons protestants venus de différents pays d'Europe (Pays-Bas, France, Autriche, etc.). Les colons aménagèrent les terres conquises comme ils savaient le faire chez eux et commencèrent à exercer une influence considérable, notamment culturelle, sur leurs voisins, avant tout sur la Pologne et la Lituanie.

Le nouveau château des chevaliers a été nommé « la montagne royale ", probablement en l'honneur du roi Ottokar II de Bohême, qui était alors à la tête de la croisade en Prusse. À côté du château ont été construits trois bourgs ayant des droits municipaux : Altstadt, Löbenicht et, de l'autre côté de la rivière, sur l'île, Kneiphof. L'année de la naissance d'Emmanuel Kant (1724), ces anciennes villes furent unifiées et devinrent Königsberg. En 1457, la résidence du grand maître de l'ordre teutonique a été transférée de la forteresse de Marienburg à Königsberg. À partir de là, la ville a acquis le statut de capitale : en 1525 elle est devenue la capitale du duché de Prusse (suite à la transformation de l'État monastique des chevaliers teutoniques en État laïc), et dès la création au $17^{\mathrm{e}}$ siècle de l'État de Brandebourg-Prusse, elle s'est transformée en résidence des monarques et en un lieu de couronnement (en 1701, couronnement du premier roi de Prusse Frédéric $1^{\mathrm{er}}$, en 1861 du roi Guillaume $1^{\mathrm{er}}$ qui est devenu dix ans plus tard le premier empereur d'Allemagne) ${ }^{1}$.

7 L'histoire de Königsberg est truffée de noms d'hommes éminents à l'échelle nationale et mondiale, dont la naissance, la vie et l'activité sont liées à cette ville. Ce sont, avant tout, des hommes de science et de culture célèbres : les philosophes Emmanuel Kant, Johann Georg Hamann, Johann Gottfried von Herder, les écrivains Johann Christoph Gottsched, Theodor Gottlieb von Hippel, Ernest Theodor Amadeus Hoffmann, les peintres Käthe Kollwitz et Lovis Corinth, les savants naturalistes Friedrich Wilhelm Bessel, Karl Ernst von Baer, Hermann von Helmholtz, Karl Friedrich Burdach, Gustav Kirchhoff, Franz Ernst Neumann ou David Hilbert. L'université de Königsberg (Albertina), créée en 1544 à l'initiative du dernier grand maître de l'ordre teutonique et premier duc de Prusse Albert de Brandebourg, fut d'une importance essentielle pour le développement de l'instruction, de la science et de la culture de la ville. 


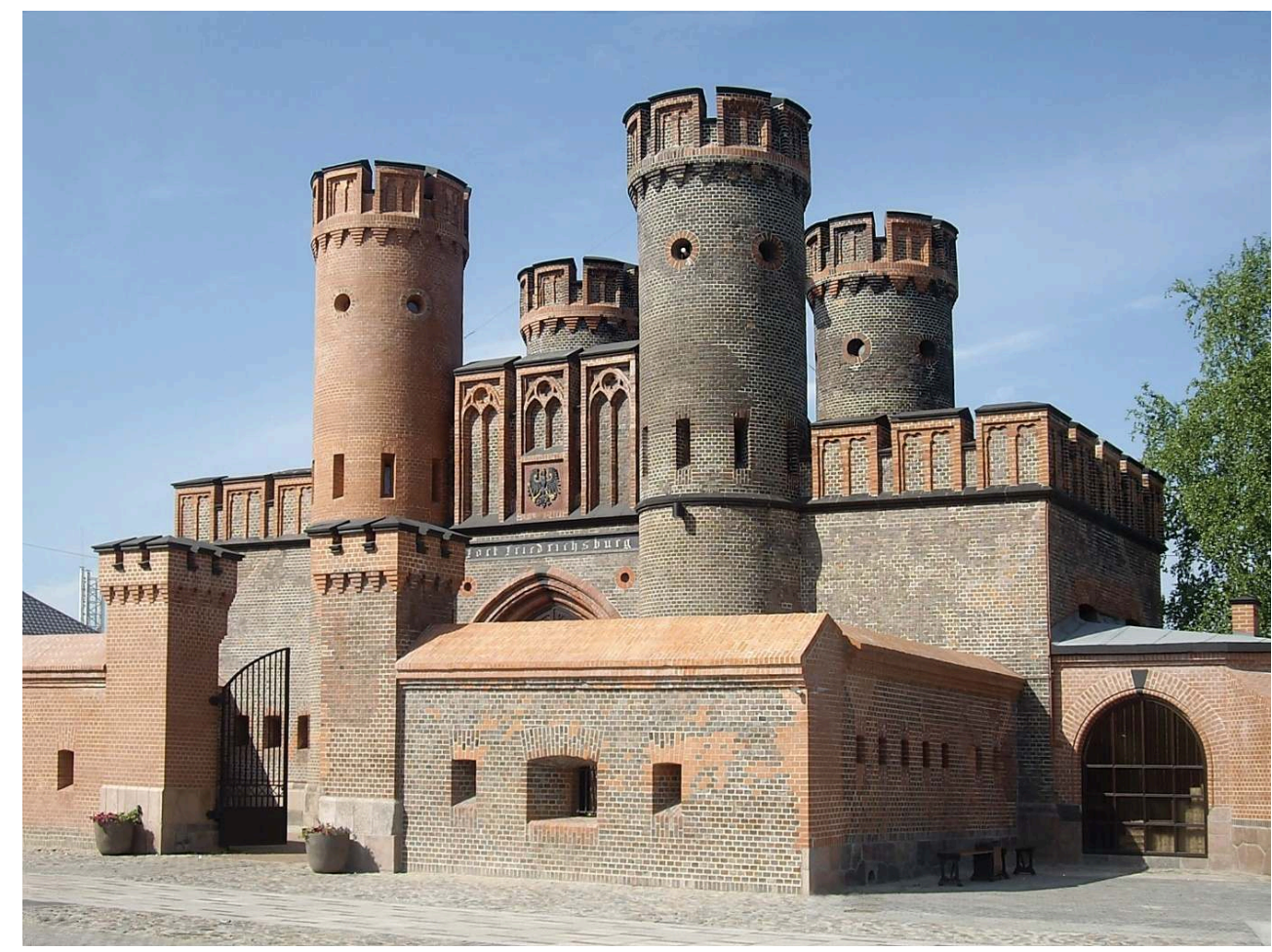

II s'agit au départ d'une construction de Friedrich August Stüler (1800 - 1865), érigée de 1852 à 1858 Photographie Yuri Bardun

Deux circonstances ont marqué son apparence en lui donnant un aspect particulier, le fait qu'elle était initialement destinée à servir à la conquête des terres païennes (comme ville-forteresse) et la proximité de la mer, lui donnant un caractère commercial (dès 1340, elle s'est jointe à l'union hanséatique). C'est pourquoi Königsberg était semblable par bien des aspects à d'autres villes allemandes sur la mer Baltique, comme par exemple Danzig. Grâce à l'esprit d'ordre et d'économie allemand, beaucoup de valeurs matérielles et spirituelles ont été accumulées dans la ville en sept siècles. Sur le plan architectural se détachaient les ensembles des trois anciens bourgs, où étaient préservés des modèles de l'architecture du $13^{\mathrm{e}}$ au $18^{\mathrm{e}}$ siècle. Parmi les bâtiments ayant une valeur particulière, il y avait entre autres le château, la cathédrale de Kneiphof (14 ${ }^{\mathrm{e}}$ siècle), l'église, devenue luthérienne, de Saint-Nicolas sur le Steindamm (la plus ancienne de la ville, datant de 1256), l'hôtel de ville de Kneiphof (fin $\mathrm{du} 17^{\mathrm{e}}$ siècle), la vieille église luthérienne de Rosgarten $\left(17^{\mathrm{e}}\right.$ siècle), quelques bâtiments d'habitation des $17^{\mathrm{e}}$ et $18^{\mathrm{e}}$ siècles, l'église luthérienne de Löbenicht de 1776 et d'autres constructions. Un autre ensemble architectural précieux était constitué par le quartier des anciens locaux de stockage sur le bord de la Pregel, ainsi que par l'ensemble des fortifications et des portes municipales. Parmi les nombreuses sculptures qui se trouvaient en ville, il faut évoquer la statue du prince-électeur de Brandebourg Frédéric III érigée en 1698 par le célèbre sculpteur allemand Andreas Schlüter dans le style baroque, qui était considérée comme le meilleur exemple de cet art à Königsberg.

9 Jusqu'au milieu du $20^{\mathrm{e}}$ siècle, le destin fut favorable à Königsberg, les quelques guerres sur le territoire de la Prusse-Orientale (y compris l'occupation russe de 1758-1762 pendant la guerre de Sept Ans) n'ayant pas fait de dégâts dans la ville. La catastrophe a commencé à la fin de la Seconde Guerre mondiale. La ville a été détruite en trois étapes. 
En août 1944, à la suite de deux puissants bombardements de l'aviation britannique, la partie centrale historique de Königsberg s'est transformée en ruines. En avril 1945, pendant l'assaut des troupes soviétiques, le centre de la ville ainsi que les quartiers périphériques ont subi des dommages encore plus graves (d'après les statistiques de 1948, sur 4896 bâtiments du centre de la ville, seulement 161 maisons sont restées intactes, c'est-à-dire seulement $3 \%$ ). Si l'on fait la comparaison, on peut constater que Königsberg n'a pas été beaucoup plus ravagée que les autres grandes villes allemandes pendant la guerre. Mais après, et jusque dans les années 1980, toutes les ruines de la ville ont été sciemment rasées (alors qu'une grande partie pouvait tout à fait être sauvée, ce dont témoigne l'expérience de la reconstruction par les Polonais de la Danzig voisine), et les maisons qui ont été restaurées ou conservées, ont en général changé d'aspect.

Durant les années de l'après-guerre, l'attitude à l'égard des restes de Königsberg a peu à peu changé. Dans les premiers mois qui ont suivi la conquête de la ville, d'avril à août 1945 (jusqu'à la conférence de Potsdam), Königsberg était considérée par le commandement militaire soviétique comme un territoire occupé. C'est pourquoi l'on avait établi un régime d'occupation sévère pour la population locale restante; le matériel technique et l'équipement des entreprises ainsi que d'autres richesses matérielles et culturelles étaient exportés en tant que trophées (il restait encore dans la ville un nombre considérable d'objets en provenance des musées, des bibliothèques, des palais ou des églises); eut lieu alors un pillage des richesses et une destruction injustifiée des maisons et des monuments culturels.

11 Avec la création en 1946 de la région de Kaliningrad et le changement de nom de la ville commencèrent le déblaiement des ruines et la reconstruction des maisons épargnées. Les ruines de la partie centrale et historique de la ville furent utilisées comme matériaux de construction, dont la plus grande part servit à reconstruire des villes soviétiques. Jusqu'au milieu des années 1950, les autorités soviétiques ont cultivé dans la population une attitude hostile envers Königsberg, qui était considérée comme la tanière d'un ennemi détesté. Vers 1949, l'architecte en chef D. Navalikhine élabora un plan de reconstruction et de développement de Kaliningrad, dont les idées principales rappelaient le plan général d'aménagement et de reconstruction de Moscou en 1937 : au centre, à la place du château royal, il y avait la majestueuse Maison des Soviets, d'où partaient, comme les rayons d'une étoile, de larges avenues, et dans les intersections des avenues avec les rues de ceinture se trouvaient des immeubles géants à l'instar des tours du Kremlin. Dans ce plan, il n'y avait pas de place pour Königsberg. 


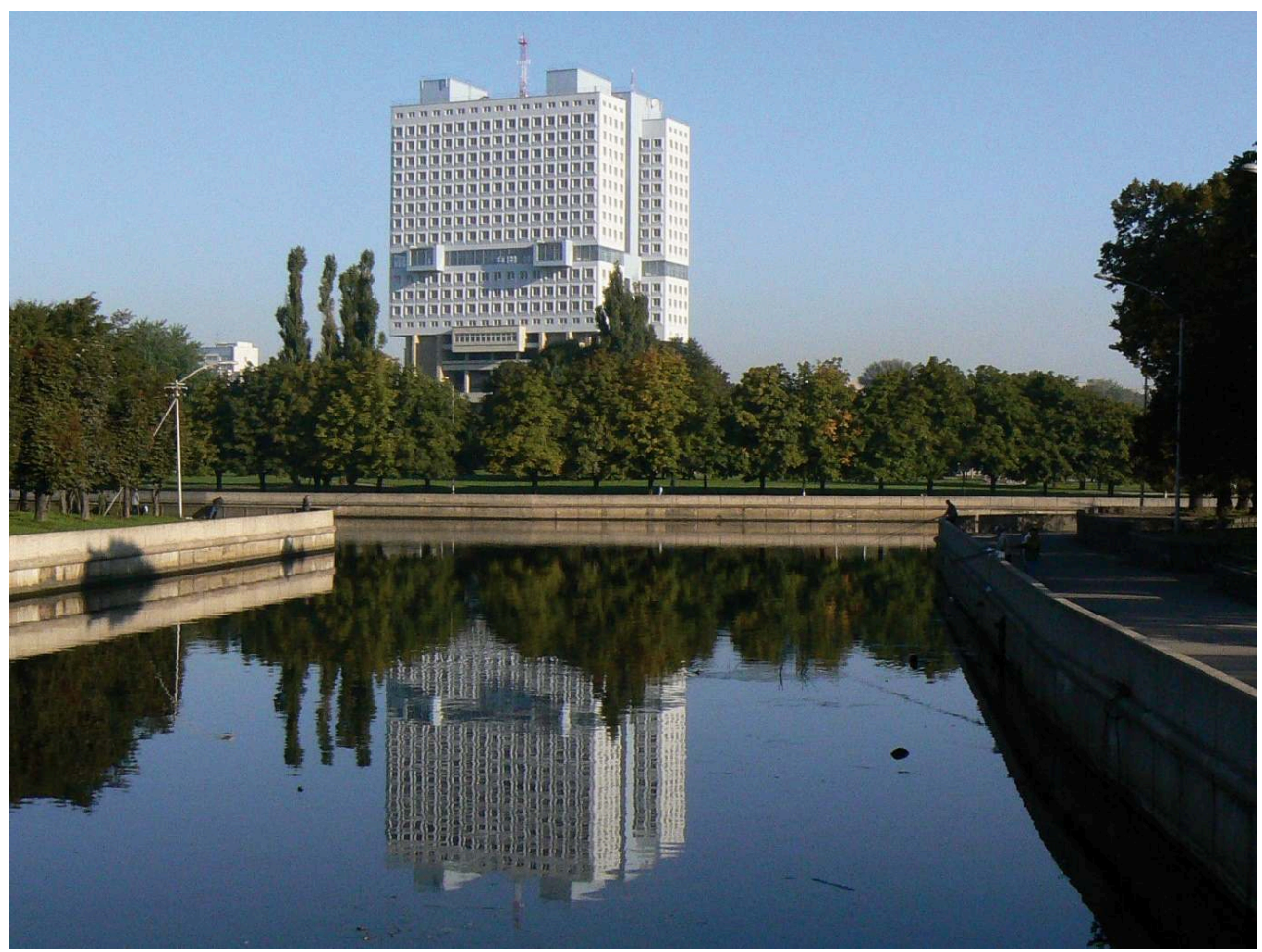

Construite dans les années 1960, elle est située sur le site de l'ancien château de Königsberg

Photographie Yuri Bardun

Dans la deuxième moitié des années 1950, sous l'influence du « dégel », on commença à réévaluer l'héritage allemand non seulement au sein de la population de Kaliningrad, mais aussi chez les représentants du pouvoir. Si en 1949 il n'y avait sur la liste des monuments historiques protégés du passé allemand que le tombeau de Kant près des ruines de la cathédrale, sur la liste de 1957, il y avait aussi les portes municipales épargnées, quelques vieilles églises, les ruines de la cathédrale et celles de la Bourse, ainsi que la statue de Schiller.

Les débats, au début des années 1960, sur le destin ultérieur des ruines du château - le symbole de Königsberg, qui pendant la période de l'après-guerre était considéré comme l'expression de «l'esprit prussien » détesté - ont constitué le moment décisif dans la réévaluation des monuments de l'ancienne ville. Cette discussion avait été initiée en 1961 par le nouvel architecte en chef de la ville, V. Khodakovsky, qui avait publié un article dans le journal Kaliningradskaya pravda, dans lequel il proposait de conserver les ruines du château en vue de leur transformation ultérieure en « un monument contre les guerres dévastatrices ». En 1963, l'architecte proposait son projet de restauration et de transformation du château en «maison populaire" avec des salles pour la réalisation d'actions diverses et un musée consacré à la prise de Königsberg. Une telle proposition était devenue possible non seulement à cause du «dégel » politique, mais encore parce que l'idée principale du plan de 1949 incluant la construction de la Maison des Soviets avait perdu sa base idéologique; en 1957, dans le contexte de la déstalinisation, le plan de la construction du Palais des Soviets à Moscou avait de même été définitivement abandonné. 

approuvé par le ministère de la Culture, qui justifiait la préservation du château par le fait qu'il était lié non seulement "à l'histoire du peuple allemand, mais aussi à certains événements importants dans la vie de l'État russe ", par exemple le séjour de Pierre le Grand et la prise d'assaut de la ville par l'armée rouge. Cependant les chefs du parti à Kaliningrad insistaient sur la démolition des ruines du château et la construction d'un nouveau centre socialiste de la ville. L'arrivée au pouvoir de L. Brejnev d'une part, et d'autre part la campagne pour la protection du château, organisée par des intellectuels de Kaliningrad qui lancèrent l'appel « Gardons pour l'histoire! » dans le Journal littéraire du 30 octobre 1965, poussèrent les leaders du parti à une action décisive. En décembre 1965, le secrétaire du comité régional du parti N. Konovalov annonça lors d'une conférence des communistes que Brejnev soutenait l'idée de la démolition du château. Aussitôt les premiers travaux à l'explosif commencèrent dans les ruines du château qui fut démoli de fond en comble en 1969. La construction de la Maison des Soviets commença aussitôt à son emplacement.

L'histoire de la démolition du château de Königsberg est significative pour la compréhension des problèmes du patrimoine historique et culturel de Kaliningrad. L'intervention des représentants de l'intelligentsia (architectes, écrivains, hommes de science) pour la protection du château a joué un rôle non négligeable dans le processus d'auto-identification des Kaliningradois, bien qu'elle ait eu aussi des répercussions négatives sur le destin de certains d'entre eux et qu'elle ait conduit en outre à la fermeture de la faculté d'histoire de l'université de la ville.

Kaliningrad a commencé à se couvrir de nouveaux bâtiments de façon réellement systématique seulement après la signature en 1970 de l'accord soviéto-germanique sur la reconnaissance des nouvelles frontières de l'après-guerre. A commencé alors la construction massive de grands blocs d'habitation standardisés refusant tout principe historiciste, mais sans hostilité non plus envers les fragments épargnés de l'ancienne Königsberg. Pendant la guerre, les quartiers assez vastes des villas de la fin du $19^{\mathrm{e}}$ et de la première moitié du $20^{\mathrm{e}}$ siècle disposés au nord, au nord-ouest et à l'ouest de la ville, étaient ceux qui avaient le moins souffert des ravages. La construction massive de l'époque soviétique ne les a presque pas touchés, mais les vieilles maisons qui manquaient de l'entretien nécessaire vieillissaient vite. D'autre part, l'aspect du centre de la ville et des quartiers adjacents, où avait eu lieu le plus de destructions, avait entièrement changé. De plus, le noyau historique de Königsberg (la colline qui abritait le château et le territoire des trois anciens bourgs) avait été définitivement déblayé de ses ruines et, n'ayant fait l'objet d'aucun nouveau chantier, transformé en zone de parc. Par contre, dans les quartiers proches du centre, des « boîtes habitées » impersonnelles de quatre à cinq étages furent bâties.

La Revue de la BNU, 5 | 2012 
Tombe d'Emmanuel Kant sur le côté nord-est de la cathédrale de Königsberg (Stoa Kantiana)

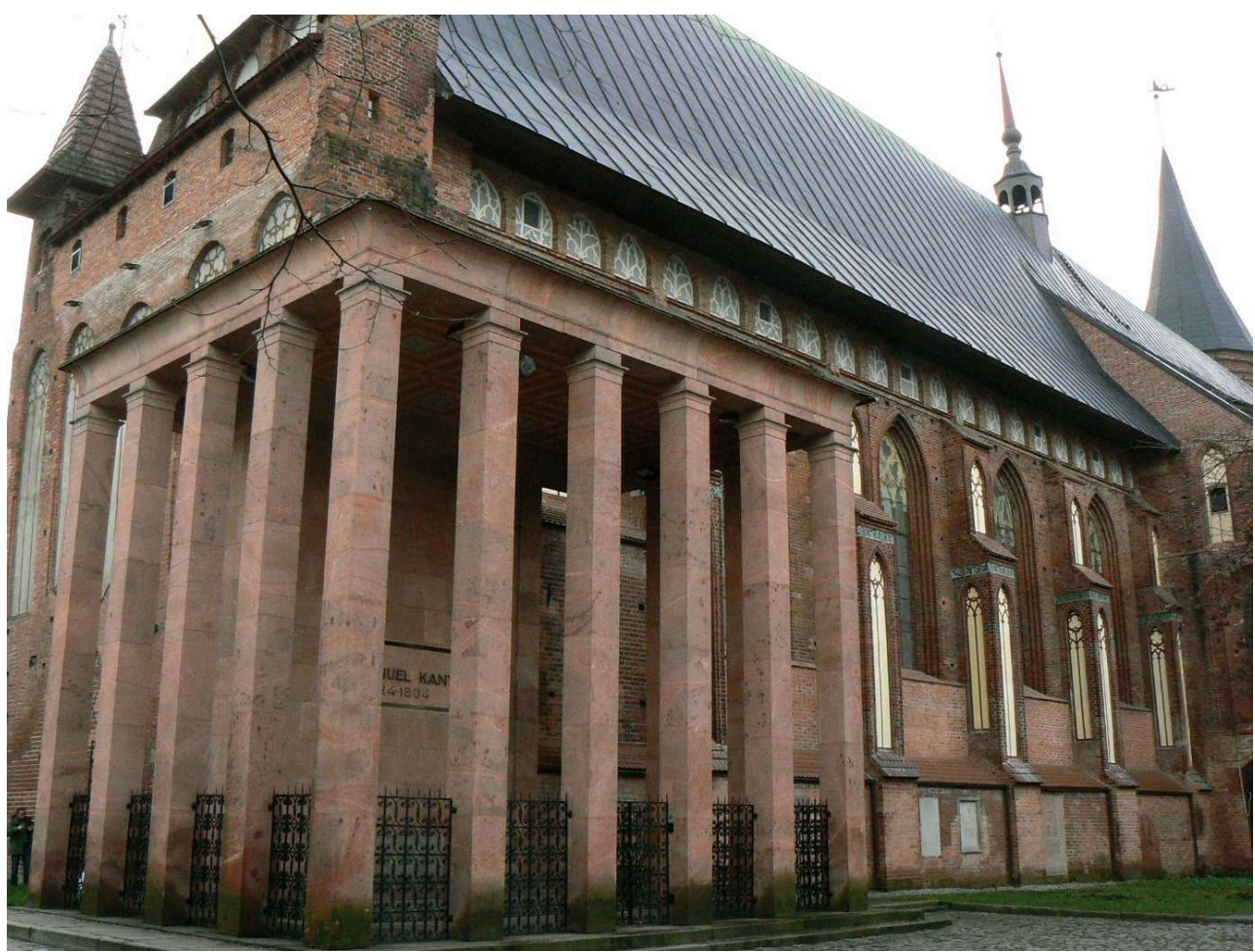

Kant fut tout d'abord enseveli dans la " crypte des professeurs », qui dès 1809 fut transformée en un passage qui reçut le nom de Stoa Kantiana (portique de Kant). Le 21 novembre 1880, les restes du philosophe furent transférés dans une chapelle de style néogothique accolée à la cathédrale. En 1924, pour le $220^{\mathrm{e}}$ anniversaire de sa mort, l'architecte Friedrich Lahrs construisit cette colonnade ainsi qu'un cénotaphe. La restauration de la cathédrale de Königsberg a débuté en 1996 par celle de la tombe de Kant

Photographie Yuri Bardun

L'attitude envers les restes de l'architecture allemande qui se trouvaient dans les zones de construction massive se distinguait par l'absence quasi totale d'expertise de la valeur historique et culturelle. Le pragmatisme et l'idéologie étaient prédominants - on restaurait seulement les bâtiments les plus grands et les plus utiles pour les autorités et les services (les bâtiments de la Bourse et de la gestion postale, un nouveau théâtre dramatique, le siège de la police, etc.), tandis que les bâtiments de culte étaient détruits ou utilisés en tant que bâtiments de stockage, salles de sport, cinémas ou clubs.

Des changements importants dans le rapport aux monuments du passé d'avant-guerre se sont produits dans les années de la perestroïka et de la période postsoviétique. Les événements moteurs de cette évolution furent la chute du diktat idéologique du parti et l'abandon de l'interdiction de l'étude et de la popularisation de l'histoire allemande de la région, ainsi que l'ouverture de la ville et de la région aux étrangers en 1990. La reconstruction de la plus ancienne église luthérienne de Kaliningrad, celle de Juditten (1280) et la consécration du premier temple orthodoxe d'après-guerre qui a eu lieu en 1985, le début de la reconstruction en 1992 de la cathédrale qui devait devenir un ensemble de musées et un centre spirituel et culturel (à présent la cathédrale est restaurée et on y trouve, entre autres, une copie de l'ancien grand orgue baroque; le tombeau du duc Albert est, lui, en cours de restauration), l'installation en 1992 à côté de l'université de la copie de la statue de Kant, qui avait disparu après la guerre et avait été restaurée en Allemagne par les efforts de la célèbre journaliste et figure publique, la 
comtesse Marion Dönhoff, toutes ces actions sont autant d'événements symboliques dans la résolution des problèmes du patrimoine historique et culturel de la ville.

Le changement de la position géopolitique de la région après la désagrégation de l'Union soviétique en 1991 et les nouveaux repères idéologiques apparus à la fin du $20^{\mathrm{e}}$ siècle ont changé considérablement l'aspect de Kaliningrad. Sur la place principale de la Victoire, en guise de " centre idéologique ", au lieu du monument de Lénine, trône l'immense cathédrale du Christ sauveur avec ses coupoles dorées et la colonne triomphale de marbre au milieu de la place. L'activité intense de l'Eglise orthodoxe russe à Kaliningrad sous la conduite du métropolite Kirill (le patriarche actuel) a suscité pour la première fois l'apparition dans la ville d'éléments de l'architecture russe traditionnelle: en plus de la nouvelle cathédrale, plusieurs autres églises ont été construites dans un style traditionnel. Parallèlement, on vit aussi une augmentation des constructions de lieux de culte des autres confessions - évangélique, catholique, néo-apostolique, arménienne-grégorienne, construits selon les traditions architecturales du christianisme d'Europe occidentale et du christianisme oriental.

Le boom architectural actuel a vu se renforcer l'accent cosmopolite dans les styles de construction employés actuellement, avec l'utilisation de motifs du vieux Königsberg. Cela s'est manifesté le plus vivement dans la construction au bord du fleuve de l'ensemble ethnographique, artisanal et commercial appelé «le village des pêcheurs ", au style néo-médiéval. La tendance à redonner une apparence historique à la ville a été réactivée lors de la préparation de l'anniversaire de Königsberg-Kaliningrad. Il a fallu remettre en état certains des monuments les plus importants de la vieille ville. Dans un temps record, on a restauré le symbole de l'anniversaire - la porte royale avec les sculptures représentant le fondateur de Königsberg, le roi Ottokar II de Bohême, le duc Albert et le roi Frédéric $1^{\mathrm{er}}$. Par ailleurs, la sculpture du grand-maître Siegfried von Feuchtwangen sur la façade de la porte de Friedland a été restaurée elle aussi; une copie de la statue perdue du duc Albert a été érigée à côté de la cathédrale, à l'emplacement du vieux bâtiment de l'université ; et enfin, un monument a été érigé en l'honneur de Liudvikas Reza, professeur et recteur de l'Université Albertina. Les autorités discutent actuellement de la question de la reconstruction du Château royal et du Kneiphof médiéval. Il était difficile de seulement imaginer de tels changements d'orientation dans les projets des autorités municipales et régionales dans un passé récent. Toutefois ces nouvelles actions sont souvent liées aux intérêts du moment et cela provoque parmi les citadins non seulement des discussions, mais aussi des protestations. 


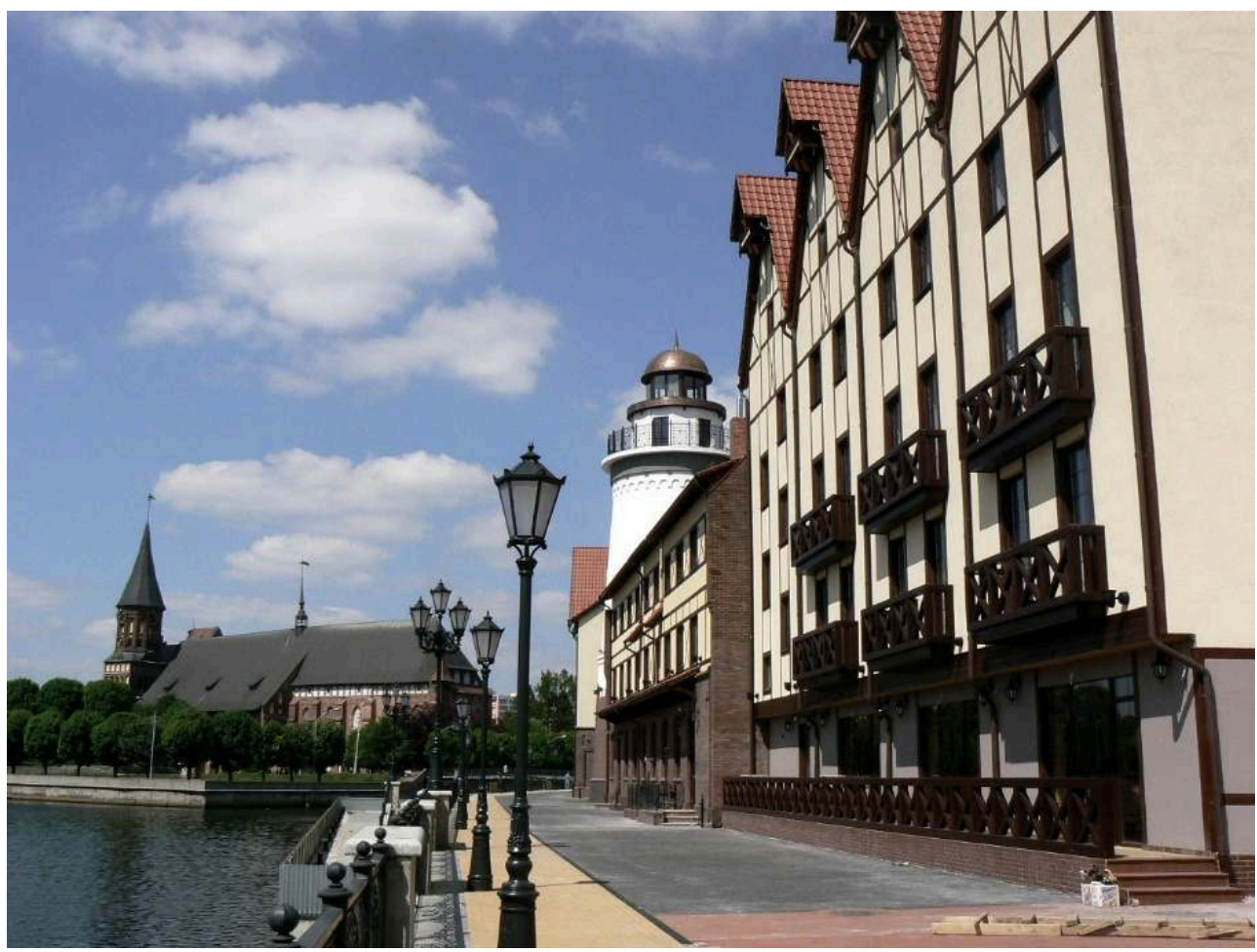

Photographie Yuri Bardun batailles politiques et idéologiques (par exemple, les discussions périodiquement renouvelées, mais toujours infécondes sur la restitution de l'ancien toponyme Königsberg), qui influencent d'une manière parfois bizarre la formation de la conscience historique des habitants de Kaliningrad.

Emmanuel Kant occupe une place importante dans le processus d'identification à l'histoire culturelle de Königsberg. On pourrait même dire qu'il est en quelque sorte un «ange gardien » de l'ancienne cité. En effet, c'est grâce au tombeau de Kant près du mur de la cathédrale, qui a été protégé comme un objet du patrimoine culturel par le pouvoir soviétique en 1945, qu'on n'a pas démoli les ruines de l'édifice, ce qui a rendu possible sa renaissance à la fin du $20^{\mathrm{e}}$ siècle. Aujourd'hui c'est non seulement un important objet culturel, mais aussi l'un des principaux symboles de l'histoire d'avantguerre et de la culture de la région qui unissent les intellectuels de Kaliningrad. Le nom de Kant a été associé en 2005 à l'Université fédérale balte créée en 1967 sur la base de l'Institut pédagogique de Kaliningrad qui, lui, avait été fondé en 1947. L'université est l'un des principaux centres russes d'études kantiennes; on y trouve la Société scientifique kantienne et l'Institut Kant, où l'on étudie et publie les travaux du grand philosophe. Par ailleurs, les scientifiques de l'université étudient et popularisent l'histoire de l'Albertina. La célébration commune, russogermanique, de l'anniversaire des 450 ans de l'université, qui a eu lieu en 1994 à Kaliningrad, est devenue un événement majeur dans ce contexte. Le professeur de l'université K. K. Lavrinovitch avait publié à cette occasion la première étude monographique, dans l'historiographie russe, sur l'histoire de l'Albertina. Le musée de l'université conserve un petit fragment de la célèbre bibliothèque de Wallenrodt, qui faisait partie de la Bibliothèque nationale 
et universitaire de Königsberg avant la guerre. Ce sont 291 ouvrages du $16^{\mathrm{e}}$ au $18^{\mathrm{e}}$ siècle, qui ont été transmis à l'université en 1981 par la bibliothèque du sanatorium de l'Académie des sciences qui se trouve à la périphérie de Moscou².

De façon générale, l'histoire de la région avant 1945 intéresse beaucoup les Kaliningradois, ce qui fait évoluer petit à petit leurs sentiments envers l'héritage allemand, sentiments qui vont de la neutralité au respect, et même parfois jusqu'à la vénération. Sur cette base se forme un patriotisme local souvent rempli de paradoxes et de mythes. Autrement dit, une difficile prise de conscience de l'implication dans le patrimoine historique et culturel d'avant-guerre et dans le destin des gens expulsés de cette terre est en marche, stimulée par la position unique de cette région russe à l'intérieur de l'Union européenne.

\section{NOTES}

1. L'histoire de Königsberg a donné lieu à une riche historiographie; parmi les études monographiques récentes se détache le travail en trois volumes de Fritz Gause, Die Geschichte der Stadt Königsberg in Preussen. - Köln-Wien, 1965-1971. En 1994 ce travail a été publié en russe en un volume, et en 1996 a paru la deuxième édition allemande.

2. À ce jour, plusieurs milliers de livres anciens provenant de Königsberg ont été trouvés dans les bibliothèques russes, $\mathrm{y}$ compris près de 2000 en provenance de la bibliothèque de Wallenrodt (cf. V. J. Kurpakov, Das Schicksal der Königsberger Bücher in der Sowjetunion nach 1945. Zu den russischen Expeditionen in das Königsberger Gebiet und den Beständen Königsberger Provenienz in den Moskauer Archiven und Bibliotheken, in Königsberger Buch-und Bibliotheksgeschichte, Köln, 2004, p. 449-468).

Comme on le sait, les livres et les manuscrits des bibliothèques les plus précieuses de Königsberg - la bibliothèque municipale, l'universitaire et celle du château (dont faisait partie la célèbre «Bibliothèque argentée » du duc Albert de Brandebourg) - ont été partiellement détruits en 1944-1945, mais la majeure partie de ces fonds s'est retrouvée dans des dépôts et des bibliothèques diverses, particulièrement en Russie, en Biélorussie, en Lituanie, en Lettonie et en Pologne (cf. K. Garber, Königsberger Bücher in Polen, Litauen und Russland, in Nordost Archiv. Zeitschrift für Regionalgeschichte. Neue Folge, Band IV, Heft I, Lüneburg, 1995).

$\mathrm{Au}$ cours des vingt-cinq dernières années, la bibliothèque de l'Université fédérale balte Emmanuel Kant a reçu plus de 5000 ouvrages en provenance des bibliothèques de Königsberg. Ce sont pour l'essentiel des donations des bibliothèques russes et allemandes. Pour différentes raisons, ni l'université de Kaliningrad, ni sa bibliothèque ne pouvaient devenir les héritières de l'Albertina et de sa bibliothèque, et c'est pour cela que le travail de rassemblement des livres de la bibliothèque universitaire de Königsberg n'a pas été commencé plus tôt. 


\section{AUTEURS}

\section{VALERIY GALTSOV}

Doyen de la Faculté d'histoire à l'Université fédérale baltique Emmanuel Kant de Kaliningrad 